\title{
Analysis of Signal Words in Chinese College Students' Argumentative Writings
}

\author{
Dongmei Sheng \\ Foreign Language Department \\ Jilin Business and Technology College \\ Changchun, China \\ 455635403@qq.com
}

\begin{abstract}
The aim of this study is to search for the best teaching method to help the college students in their argumentative writing. This paper presents an analysis of the connection words in Chinese college students' argumentative writings and examines the mistakes of the connection words. The result shows that the main reasons of the mistakes are attributed to the transfer of mother tongue and over generalization. The result suggests that the better teaching method in college students' argumentative writing is of great significance in improving college students' writing ability.
\end{abstract}

Keywords-Signal word; cohesion; coherence; argumentative writings

\section{INTRODUCTION}

Recently, writing, as one of the important four skills, attracts many people's attention. A lot of experts and scholars spent a lot on the study of writing from words, sentences until semantic model .Although there are some achievements in writing, we can still find that coherence in writing is still a big problem for Chinese students, among which the use of signal words is one of them. As is well-known, Chinese and English belong to different language families, and it is only natural that they may pose great difficulties and challenges for beginners such as my students [1]. The formal and serious teaching of cohesion might to a great extent enhance the students' consciousness in writing the argumentation. These transitional words are some conjunction and link adverb, indicating respectively the relation of time and space, relation of extending and transition, relation of concession, relation of list and illustration.

\section{Definition OF FIVE Types OF COHESION}

According to the semantic relationship, classification of Halliday and Hasan's connection are supplement, causality and turning and time [2]. This study connects words according to the meaning of the division in the following several types: lists, supplement, cause and effect, turning or contrast In this paper, through the analysis of English Majors' argumentative writing in misuse of conjunctions and the reasons, the writer hope that this paper can help the teachers in English teaching and offer some suggestions for them As a kind of connection between the two different languages, the connection word (conjunction) is one of the biggest differences between English and Chinese. In the text, the language component is realized by means of five means such as the substitution, the substitution, the vigor, the connection and the lexical cohesion. As one of the means of discourse cohesion, coherence of text link plays an important role. By connecting the word, the reader can understand the semantic relations between sentences after the preview. Thus, many researchers with different cultural backgrounds may understand these linking words.

Five types of cohesion are ellipsis, substitution, conjunction and lexical cohesion. The connection element performs cohesion function. Through the utility of connection word, people not only build up semantic connection of sentences, but also predict the follow -up semantic from logic. Reference is a device which allows the reader or hearer to trace participants, entities, events, etc. in a text. Substitution and ellipsis, unlike reference, are grammatical rather than semantic relationships. In substitution, an item is replaced by another item: For example: Do you like movies? I do. In the above example, do is a substitute for like movies. Items commonly used in substitution in English include do, one, and the same.

Ellipsis involves the omission of an item. In other words, in ellipsis, an item is replaced by nothing [3]. This is a case of leaving something unsaid which is nevertheless understood. It does not include every instance, in which the hearer or reader has to supply missing information, but only those cases where the grammatical structure itself points to an item or items that can fill the slot in question. Here is an example: Joan brought some carnations and Catherine some sweet peas.

Lexical cohesion refers to the role played by the selection of vocabulary in organizing relations within a text. A given lexical item cannot be said to have a cohesive function per se, but any lexical item can enter into a cohesive relation with other items in a text. In passages, cohesion mainly depends on the connection between sentences and the element on each function according to its own meaning. Also, with the help of transitional words, people can not only establish the semantic connection between sentences but also predict the semantic meaning of the following sentences logically. Scholar did a research on the use of cohesion theory and found that the favorite ways students' use is: words, logical connector and., which on the other hand has shown that logical connector has been paid more attention in teaching. 


\section{ClassificAtion OF LOGICAL CONNECTOR}

Logical connector, also called signal words, is the more complicated one in linking up the means. It labels a kind of relation that can only pass and totally understand the passages according to other parts. Logical connector, as one of the transitional means decides the development of passages, shows the logical relationship between words, sentences and paragraphs. Without proper transitional means, the content of the passage can't be fully understood.

Conjunctions, adverbial conjunctions, prepositions and prepositional phrases, as Quick said, belong to logical connector [4]. Functioning as a bridge, these transitional words and phrases connect the passages logically, express the papers naturally, and show the relationship between each part clearly. Signal words, as Adam call them, signal the natural transition from this idea to that one and give readers the direction and the clue of the sentence development. Without these words in the relating sentences, cohesion and coherence can't be found. So the signal words are used to show the logical relationship, signal the occurrence of some semantic concepts and thus these words themselves are used to

In form, logical connector consists of words, phrase and words; in meaning, they can show some types of semantic relations. Although many linguists show the ways of classification without agreement, we know that logical connector expresses different semantic meaning. The following is the common classification:

- Supplement and, also, too, in addition, moreover, besides, furthermore, not only---but also, above all, as well as, together with, along with, next, what's more。

- Time after, before, soon, when, until, during, immediately, then。

- Example for example, for instance, such as, that is, in other words, in fact。

- Similarities like, as, similarly, in the same way, as well, too, equally, likewise。

- Contrast: unlike, in contrast with/to, whereas, on the contrary, on the other hand,

- Cause and effect: since, because, due to, owing to, for, as, because of, on account of, as a result,

- Purpose: in order to, in the hope that, so as to, so that, in the hope of

- Summary: in a word, in conclusion, on the whole, in short, to conclude, to sum up, finally, at last

- Sequence: first, then, next, after that, finally, last of all, at the beginning, one ,another, still, soon, later, initially

- Condition: if, unless, on condition that, as long as, in case, suppose

- Space: above, across, beyond, in front of , next, adjacent to, to the left

- Emphasis: chiefly, especially, indeed, in fact, actually

\section{USE OF LOGICAL CONNECTOR IN WRITING}

Although teachers and students have more and more realized the importance of transitional means, there are still some problems that can be found from students' paper. Take one of the papers Campus Love for example. The following is the improper use of logical connector found in the fifty students' paper.

\section{A. Improper Use of Logical Connector}

Because of misunderstanding the meaning, for example: on the contrary/ In contrast, conjunction involves the use of formal markers to relate sentences, clauses and paragraphs to each other. Unlike reference, substitution, and ellipsis, the use of conjunction does not instruct the reader to supply missing information either by looking for it elsewhere in the text or by filling structural slots. Instead, conjunction signals the way the writer wants the reader to relate what is about to be said to what has been said before. Conjunction expresses one of a small number of general relations is summarized below, with examples of conjunctions which can or typically realize each relation.

\section{B. Overuse of Logical Connector}

Students would prefer to use more logical connector to strengthen sentence links. But in fact, it will lead to the excessive use of logical connector, which in turn increase surplus information instead, even makes the logical confusion between sentences. Although it is nice to see that Chinese students begin to realize the use of transitional means, yet excessive use of logical connector is still a serious problem [5]. Experts have found that logical connector concerning example, result, and are students' favorite in their argumentation. Even some scholars, using the way to count the frequency of each kind of conjunctions by Wordsmith, examine with the card side, and then put in order through Excel record, and analyze with SPSS, find that Chinese students prefer logical connector on "supplement" and "summary" to that on "space", and in most cases in using logical connector, simple words are preferred. Chinese students are not good at using various logical connectors, which should be proper and intractable. Concise and proper use of logical connector is necessary; otherwise it will take readers' mind off.

\section{Connection in Confusion}

In English "on the other hand" and "on the contrary” is two common use of discourse marker, which is used to indicate that the semantic is relative or opposite, but they have difference between them. During the course of study, the students often are confused with their concepts [6]. For example: making friends online doesn't seem unacceptable to me, on the other hand, I think it deserves much consideration. This sentence doesn't mean two aspects of one matter, so "on the other hand "should be changed into "on the contrary "Sometimes the students will use link words as conjunction. But is a parallel connection of two parallel noun clauses and two contrasts in the general sense. But however can not directly connect two clauses and it must be separated by new words and commas. 
Some experts also pointed out those Chinese students usually tend to put it in the middle of the sentence, while the English native speakers will put this word in the first part of the sentence. In an addition, Chinese students rarely put however in the middle of the sentence for the insertion, but the English native speakers intend to put the however in the middle of the sentence. The same situation also appears in the words of so and therefore, indicating that in the course of Chinese EFL learning the target language position of conjunction in late to acquisition of the initial position. This may occur because they know a little about the usage of the word.

\section{Error of Wrong Connection Selection}

Errors in the use of connection make the syntax confused. It is obvious that some students do not choose the correct connection and lead to the wrong expression of the meaning of the sentence. For example, at the other hand, we must leave enough time to do everything. Otherwise how to arrange for time correctly is very important." Otherwise" is generally used to indicate the anti sense, but the two sentences have causeeffect relationship. If it is converted into a "for", the situation will be improved. Another example We should not ask for more from the earth and continue the endless consumption. The two negative components are connected by "or" instead of "and". Because the understanding of the English word is not comprehensive, the students will make these mistakes.

Science is the production of knowledge that can be applied or not, since technology is the application of knowledge to the production of some product, machinery or the like. In the example, before and after a comma the two sentences have a contrast relationship, but the word since makes the sentence into a causal relationship, so the whole meaning of the sentence becomes blurred.

Therefore, since should be changed into while or whereas. Even if the same semantic relationship shows the subtle differences in the different connection words and it can not be exchanged [7]. For example, in and, also, besides, what 'more s, addition in, furthermore, these words are followed by the word in front of the group in order to be more formal, stronger .Therefore, in English, the use of the word, the wide use of the complex, also become a major difficulty in student writing.

\section{E. Deficiency of Conjunction}

English and Chinese sentences can be divided into simple sentence and complex sentence. In the Chinese language without associated words can also construct sentences. In Chinese, when you want to change the topic, you do not need to connect words to another topic directly from a topic. But in English, changing the subject should have proper connectives to guarantee the syntax and semantics of the correct sentence. Therefore, the students will be easily influenced by the Chinese in the use of English connectives, resulting in negative transfer. For example: They have discussed the problem, ex-changed their opinions on it. They have not figured out these two sentences in the sense. That is, to discuss, exchange, not find the program. The logical meaning of the two sentences is more obvious if it is combined with the proper connection words.

\section{INFLUENCE OF CULTURE}

Thanks to the close relation between language and culture, it is inevitable for Chinese students to be influenced by Chinese thinking way and Chinese value in their writing. And the use of logical connector is one example of it. Sometimes, without proper use of some logical connector, some sentence with correct use in grammar and in meaning still can reflect the logical connection between sentences. For example: They (College students)can talk with each other, play with each other, help each other, learn from each other.

They can get a lot of happiness. In fact, these two sentences are connected together in meaning, namely: The happiness of love comes from talking. So if the sentence is like this: They (College students) can talk with each other, play with each other, help each other, and learn from each other, from which they can get a lot of happiness. it will be better. In changing the topic, Chinese will directly change from this one to that one; while English needs proper logical connector, or comma splice (a kind of not accepted sentence patterns in English) will be often seen. For example: The part: "Some students studies, some students go shopping on weekend" is a comma splice because of the lack of proper logical connector. The correct one should be. Some students studies, while some students goes shopping on weekend.

Chinese "parataxis", namely the semantic relationship between the sentences or paragraphs does not rely on conjunctions as a link, but through the logical relations between sentences or paragraphs, sentences sequence pay attention to the connection of implicit meaning. While English tends to "taxis hypo", that is, in most cases, it is connected with the semantic relations of the text in the form of explicit connection. Due to the difference of "meaning" and "form", the frequency of conjunction in Chinese composition is far less than that of English composition. Chinese often use a comma to connect several complete sentences. Therefore, Chinese sentences, the so-called running sentence are composed of a series of complete sentences, grammar and words loosely connected. Therefore, this sentence will continue to move to the next and be not separated into two sentences. However, English as a language of hypotaxis, must use linking words of two clauses. Chinese use commas and ignore the connectives. Many students are influenced by Chinese and there are a lot of running sentences in English writing.

Here the intervention structure mainly refers to the students in the writing who apply Chinese word. On the morphological structure, the number of single use and the number of pairs of words in Chinese are similar, while in English, the number of single use is more than that. Sometimes in order to express the same semantic relationship between English and Chinese, conjunction with paired conjunctions will be used in such situation. Such as "I have made some suggestions, but there is no response." "I put a lot of suggestions, but no results."

\section{ADVICE FOR TEACHING}

We must admit that, achieving coherence by logical connector is not all in coherence [7]. For students in elementary stage, logical connector in argumentation still needs attention. According to the problem students get, teacher should pay 
attention the following aspects: Traditional teaching focuses on words, sentences, paragraphs and even the whole passages. In learning English, it is inevitable for students to think in Chinese way and this influences their English study. So it is quite necessary for teachers to give emphasis on the culture teaching to students. Teaching logical connectors, accuracy and variety of logical connector should be the emphasis. Besides knowing the common logical connectors, more exercises should be given. Many ways are effective such as the cooperation of students to find one paragraph with good logical connector and exchange with each other, learn or recite or even imitate them.

Some experts pointed out that the frequency distribution of the connection words in the good composition was significantly higher than the frequency distribution of the difference. In the study they found that Chinese students use logical connection words more frequent than the native speaker. They also pointed out that the majority of English major graduate students did this? In the process of writing, the correct and reasonable use of the connection words can improve the efficiency of the readers, understand the intention of the text and promote the exchange of information.

Through the investigation and study, it is found that students need to improve their English writing quality, and also need to improve their comprehensive ability. English discourse connection as a transitional means, is not essential. In the teaching of English writing, teaching is connected with the usage of the word, which should help students to overcome the negative transfer of Chinese, guide students textual meaning, help them clarify ideas according to the events of different types and different context. In teaching practice, in addition to the commonly used connection words, the emphasis should be placed on the accuracy and diversity.

\section{CONCLUSION}

From learning, we get that logical connector is the third one on coherence ways, while it really plays an important role. Of course, logical connector also plays the same function between paragraphs, sentences and paragraphs, etc. which hasn't been mentioned in this paper. Nowadays, English being a hot topic has been studied by more people, and logical connector being a new subject has a long way for experts

\section{REFERENCES}

[1] Biber. Det al. Longman Grammar of Spoken and Written English.Beijing Foreign Language Teaching and Research Press, 2000.

[2] Ellis Rod. The Study of Second Language Acquisition.Shanghai Foreign Language Education Press, 1994:51.

[3] Halliday, M.A. K. \& R. Hasan. Cohesion in English. Beijing Foreign Language Teaching and Research Press,1976.

[4] Dagut, M., Laufer, B.1985. Avoidance of phrasal verbs---A case for contrastive analysis. Studies in Second Language Acquisition, 7, pp. 7379.

[5] Fraser, B. 1996. Pragmatic markers. Pragmatics, 6 (2), pp. 167-90.

[6] Fraser, B. 1999. What are discourse markers? Journal of Pragmatics, 31 (7),

[7] Fraser, B. 2005. Towards a theory of discourse markers. In K. Fischer (Ed.), Approaches to Discourse Particles, 1, pp. 189-203. Amsterdam: Elsevier Ltd. 